Spin Physics (SPIN2014)

International Journal of Modern Physics: Conference Series

Vol. 40 (2016) 1660072 (6 pages)

(C) The Author(s)

DOI: 10.1142/S2010194516600727

\title{
Polarization Observables in Few-Nucleon Scattering
}

\author{
St. Kistryn \\ Institute of Physics, Jagiellonian University \\ PL-30059 Kraków, Poland \\ stanislaw.kistryn@uj.edu.pl \\ E. Stephan \\ Institute of Physics, University of Silesia, \\ PL-40007 Katowice, Poland \\ elzbieta.stephan@us.edu.pl \\ Published 29 February 2016
}

\begin{abstract}
Systems composed of a few nucleons are subject of experimental studies for many years with the aim to gain precise information on the interaction between nucleons. Intermediate energies, below the threshold for pion production, deserve special attention: it is a region where comparison with exact theoretical calculations is possible, while the sensitivity to various aspects of interaction, like subtle effects of the dynamics beyond the pairwise nucleon-nucleon force, is significant. In addition to differential cross section, the observables related to nuclear polarization are studied, like vector and tensor analyzing powers, spin-correlation coefficients or polarization transfer coefficients. A brief survey of results of recent studies in few-nucleon systems is given.
\end{abstract}

Keywords: Few-nucleon systems; three-nucleon force; polarization; analyzing power.

PACS numbers: 24.70.+s, 25.10.+s

\section{Introduction}

In general, an exact understanding of all features of few-nucleon system dynamics would provide a natural basis for describing properties and interactions of nuclei. This knowledge is important for fundamental nuclear physics, but also for several fields of its application. The nucleon-nucleon $(N N)$ potential is undoubtedly a leading part of the nuclear interaction and should be sufficient to describe basic properties of nuclei and main trends in observables for systems of few (and many) nucleons, if only exact calculations are feasible. The $2 N$ system has been intensively experimentally studied over last decades forming a solid data base, on which

This is an Open Access article published by World Scientific Publishing Company. It is distributed under the terms of the Creative Commons Attribution 3.0 (CC-BY) License. Further distribution of this work is permitted, provided the original work is properly cited. 
modern models of $N N$ potentials have been founded. However, since the internal structure of nucleons is neglected, the question arises, how the suppressed degrees of freedom influence observables in any system consisting of more than 2 nucleons. Such additional dynamics, which cannot be reduced to pairwise forces, is referred to as three-nucleon force (3NF). The importance of $3 \mathrm{NF}$ contributions for a proper description of systems of more then two nucleons was first established in few-nucleon bound states. ${ }^{1-4}$ Studies of $3 \mathrm{~N}$ systems in nuclear reactions are mainly focused on measurements of observables for elastic nucleon-deuteron scattering and for breakup of a deuteron in its collision with a nucleon. Full dynamical information on the process can be gained by determining a so-called complete set of observables. Therefore, in addition to differential cross section the studies comprise the observables related to nuclear polarization, like vector (and tensor) analyzing powers, spin-correlation coefficients or polarization transfer coefficients. Extensive discussions of the present status of understanding of the $3 N$ system dynamics, based on the modern calculations and many precise and rich data sets, can be found in recent reviews. ${ }^{5-7}$

\section{Elastic Scattering}

When exact calculations including $3 \mathrm{NF}$ for observables in the nucleon-deuteron elastic scattering became available, the models of $3 \mathrm{NF}$ turned out to be an efficient remedy for a long persisting problem of a proper description of cross section for this process. $^{8-10}$ Though at beam energies above $100 \mathrm{MeV}$ per nucleon certain problems with describing the data are still observed (cf. Ref. 11 and references therein), improvement of description of the cross section data due to the inclusion of $3 \mathrm{NFs}$ is still considerable. On the other hand, precise experimental data demonstrate both, success and difficulties of the current models in describing analyzing powers, spin transfer and spin correlation coefficients. ${ }^{12-20}$ This indicates problems with the spin part of the models of the $3 N$ interaction. Discrepancy between the predicted and measured tensor analyzing power $T_{22}$ in the region of intermediate energies is one of the examples illustrating the problem. This observable has been extensively measured at deuteron beam energies of 100, 130 and $140 \mathrm{MeV}$ in experiments performed at a few laboratories with the use of various techniques. In Fig. 1 all the results are presented together, in terms of the net effect of $3 \mathrm{NF}$. For this purpose the $T_{22}$ values obtained via calculations with the pure CD Bonn potential ${ }^{21}\left(T_{22}^{2 N}\right)$ were subtracted from both, the data points and the predictions obtained with the inclusion of the TM99 $3 \mathrm{NF}^{22}\left(T_{22}^{3 N+2 N}\right)$. The same procedure was applied for all three beam energies: $100 \mathrm{MeV}$ (full dots and dash-dot line), $130 \mathrm{MeV}$ (open dots, ${ }^{20}$ open triangles, ${ }^{19}$ solid line) and at $140 \mathrm{MeV}$ beam energy (full triangles, ${ }^{15}$ dashed line). Below $120^{\circ}$ the predicted effects of the TM99 3NF depend significantly on energy, while at larger angles they do not reveal such feature. Behavior of the data is very different: below $120^{\circ}$ majority of the data points is consistent with zero, i.e. they are well described by the pure $N N$ force predictions. At larger angles, the departure of the data from the calculations with the pure $N N$ force rises with the beam energy. 


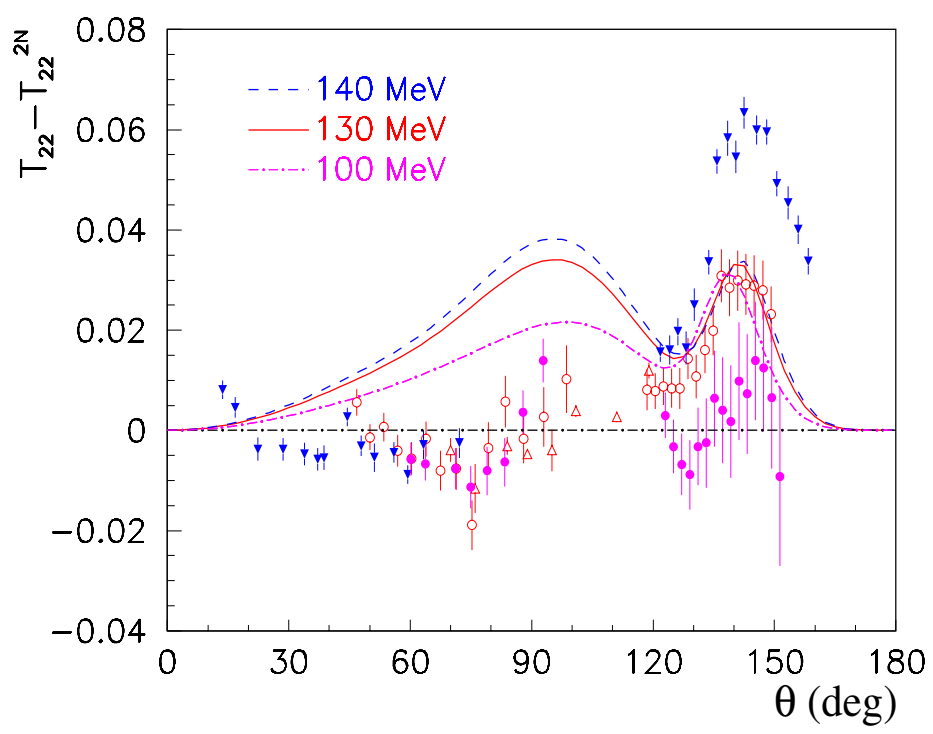

Fig. 1. Vector and tensor analyzing powers of the $d$-p elastic scattering: Net effect of $3 \mathrm{NF}$ for the $T_{22}$ data compared to the predicted effect of TM99 3NF. For more details see text.

Adding of 3NF improves the description significantly in the case of the measurement at $130 \mathrm{MeV}$, but for the beam energy of $140 \mathrm{MeV}$ the predicted effect of $3 \mathrm{NF}$ is not large enough to reproduce the data, while at $100 \mathrm{MeV}$ the predicted influence of $3 \mathrm{NF}$ is to strong (even though statistical significance of the results at $100 \mathrm{MeV}$ is not convincing). As a general remark it is worthwhile to stress that unambiguous conclusions on the quality of the data description are conditioned by precise experimental data covering large phase space and performed at several energies.

\section{Breakup Reaction}

Conclusions on the role of $3 \mathrm{NF}$ for the description of the cross section for the breakup reaction are to a large extent similar to the observations for the elastic scattering. Significance of $3 \mathrm{NF}$ for a correct description of the differential cross section has been confirmed, e.g. at $130 \mathrm{MeV} \cdot{ }^{23,24}$ In contrast to the elastic scattering, practically sensitive to neither Coulomb interaction ${ }^{26}$ nor to relativistic effects ${ }^{27}$ over a wide range of energies, the cross section for the breakup reaction reveals, due to a variety of kinematical configurations of its final state, local sensitivity to both of these effects. ${ }^{28,29}$ The Coulomb effects turned out to be surprisingly large, ${ }^{24,30}$

Polarization observables have been also studied for the breakup reaction, although the world data base is much poorer in variety of the studied observables and beam energies as compared to the elastic scattering case. It is very unfortunate regarding the potential of information inhered in the reaction: For example, "parityodd' analyzing powers, forbidden by symmetry restrictions in the elastic scattering, 
can be here studied. The first rich set of analyzing power results for the ${ }^{1} \mathrm{H}(\overrightarrow{\mathrm{d}}, \mathrm{pp}) \mathrm{n}$ breakup reaction was collected at the beam energy of $130 \mathrm{MeV}$ and encompasses two vector, $A_{x}, A_{y}$, and three tensor, $A_{x x}, A_{y y}, A_{x y}$, analyzing powers (here given in their Cartesian representation). They have been evaluated at about 800 kinematical points for each observable and compared to predictions of various theoretical approaches. ${ }^{31}$ Tests of data consistency and proofs of validity of the applied analysis methods are of crucial importance for precise experiments. A very handy test, based on parity restrictions imposed on the analyzing powers, is shown in Fig. 2. Space reflection symmetry requires that the analyzing power value extracted at a certain kinematical point defined by the proton emission angles and variable $S$ related to the proton energies ${ }^{7}\left(\theta_{1}, \theta_{2}, \varphi_{12}, S\right)$ and evaluated for the so-called "mirror" configuration point, characterized by the reversed value of the relative azimuthal angle $\varphi_{12}\left(\varphi_{12} \rightarrow-\varphi_{12}\right)$ have to be either identical (for $A_{y}, A_{x x}$ and $A_{y y}$ ) or differ in sign (for $A_{x}$ and $A_{x y}$ ). It is therefore possible to construct parity-forbidden combinations of the analyzing powers, $O_{r}=A_{r}\left(\varphi_{12}\right)+(-1)^{q} \cdot A_{r}\left(-\varphi_{12}\right)$, where $q=1$ for $r \equiv y, x x, y y$ and $q=0$ for $r \equiv x, x y$, which should all be consistent with zero. An example of $O_{x}$ for one selected angular configuration, presented as a function of $S$, is shown in the right panel of Fig. 2. The excellent consistency with zero demonstrates high quality of the evaluated analyzing power data.

It turned out that deuteron vector analyzing powers of the breakup reaction at 100 and $130 \mathrm{MeV}$ are equally insensitive to any additional dynamics beyond the pure $N N$ interactions as it has been earlier observed for the d-p elastic scattering

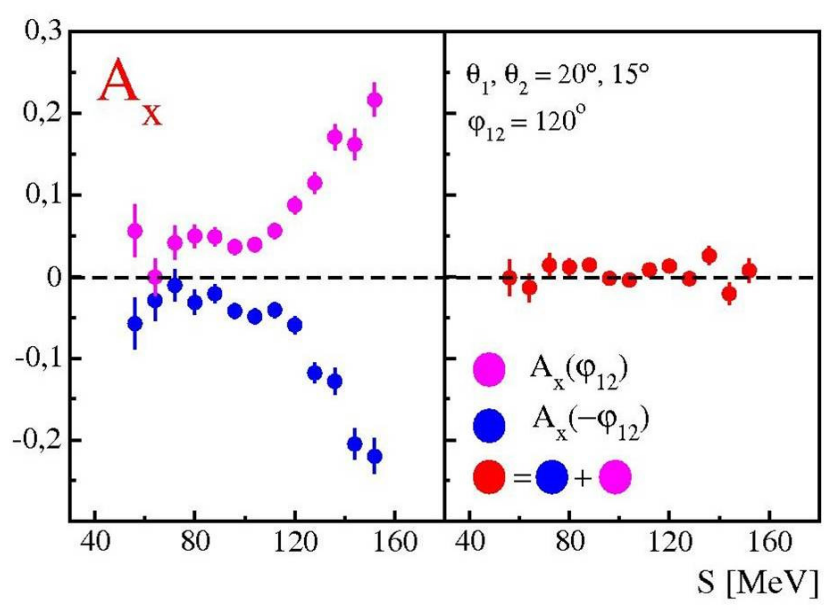

Fig. 2. Data consistency check by means of comparing the parity-forbidden combination of the analyzing power values. Left panel: Distribution of the parity-odd analyzing power $A_{x}$ at the selected kinematical configuration (red/light gray dots) and its values for the "mirror" configuration (violet/dark gray dots). Right panel: Resulting distribution of the sum of the values from left panels. 
at the same energies. ${ }^{31,32}$ On the other hand side, for the tensor analyzing powers of the breakup reaction at the same beam energies, locally certain discrepancies are observed, ${ }^{31}$ even if (or when) 3NF is included into calculations. The experimental studies of the ${ }^{2} \mathrm{H}(\vec{p}, \mathrm{pp}) \mathrm{n}$ reaction at 135 and $190 \mathrm{MeV}^{33,34}$ show that for vector analyzing power $A_{y}^{p}$ a large discrepancy between the measured data and the theoretical predictions can be observed at small relative azimuthal angles of the two breakup protons and this deficiency even increases when including 3NF. The discrepancy rises with the beam energy and the predicted relativistic effects do not explain this behavior. These facts confirm a problem with the description of spin observables in $3 \mathrm{~N}$ systems. Precise data sets for polarization observables of the ${ }^{1} \mathrm{H}(\vec{d}, \mathrm{pp}) \mathrm{n}$ at the beam energy of $270 \mathrm{MeV}$, collected at $\mathrm{IUCF}^{35}$ and at RIKEN, ${ }^{36}$ showed also "a mixed picture" in the sector of spin observables. For example, $A_{y}$ obtained in several angular configurations is described properly by the pure $N N$ force predictions, while including 3 NFs leads to a deterioration of the agreement. ${ }^{36}$ It has been found that such behavior is opposite to the one in the elastic scattering at $270 \mathrm{MeV}$.

The first attempts were made also to study experimentally $4 \mathrm{~N}$ systems. Due to the lack of precise calculations, spin observables in the three-body break-up reaction in deuteron-deuteron scattering were analyzed in the phase-space regime that corresponds to the quasi-free deuteron-proton scattering process with the neutron as a spectator. ${ }^{37}$ Such choice allows for their direct comparison to the analyzing powers of the elastic deuteron-proton scattering. One of the three studied analyzing powers $\left(T_{20}\right)$ revealed significant discrepancies, but their source is not clear it might be attributed to the final state interactions and, in consequence, to the limited applicability of the quasi-free condition assumption.

\section{Summary and Outlook}

Polarization observables provide important insight into the details of few-nucleon system dynamics. Only with their use it is possible to probe quality of spin structure of the $3 \mathrm{NF}$ models. Therefore precise data in this sector are extremely valuable and longed-for. Recently, opportunities to perform experimental studies of 3 and 4-nucleon systems in a wide range of proton beam energies opened in Poland, at the Cyclotron Center Bronowice of INP PAS in Kraków, with the use of the multidetector system BINA, successfully applied previously in a number of experiments at KVI, Groningen, The Netherlands. In the first step measurements of differential cross sections are planned, later an extension to polarization observables is also considered. The developments in theoretical description of the few-nucleon systems are ongoing, e.g. with the goal to include simultaneously various important pieces of dynamics (3NF, Coulomb interaction, relativistic effects) or to further develop calculations based on Chiral Perturbation Theory. Another direction of developments is driven by desire to perform ab-initio calculations for $4 \mathrm{~N}$ systems at intermediate energies - such a step, together with a gradually growing data base for those systems, would open a new chapter in the studies of few nucleon systems. 


\section{Acknowledgments}

This work was supported by the European Commission within the Seventh Framework Programme through IA-ENSAR (contract no. RII3-CT-2010-262010) and the National Science Center Poland founds DEC-2012/05/B/ST/02556.

\section{References}

1. M. Viviani, Nucl. Phys. A 631, 111c (1998).

2. A. Nogga, H. Kamada and W. Glöckle, Phys. Rev. Lett. 85, 944 (2000).

3. S. C. Pieper and R. B. Wiringa, Ann. Rev. Nucl. Part. Sci. 51, 53 (2001).

4. P. Navratil et al., Phys. Rev. Lett. 99, 042501 (2007).

5. K. Sagara, Few Body Syst. 48, 59 (2010).

6. N. Kalantar-Nayestanaki, E. Epelbaum, J. G. Meschendorp and A. Nogga, Rep. Prog. Phys. 75, 016301 (2012).

7. St. Kistryn and E. Stephan, J. Phys. G: Nucl. Part. Phys. 40, 063101 (2013).

8. H. Witala et al., Phys. Rev. Lett. 81, 1183 (1998).

9. K. Hatanaka et al., Phys. Rev. C 66, 044002 (2002).

10. P. Mermod et al., Phys. Lett. B 597, 243 (2004).

11. A. Ramazani-Moghaddam-Arani, et al., Phys. Rev. C 78, 014006 (2008).

12. E. J. Stephenson et al., Phys. Rev. C 60, 061001 (1999).

13. R. Bieber et al., Phys. Rev. Lett. 84, 606 (2000).

14. H. Sakai et al., Phys. Rev. Lett. 84, 5288 (2000).

15. K. Sekiguchi et al., Phys. Rev. C 70, 014001 (2004).

16. K. Ermisch et al., Phys. Rev. Lett. 86, 5862 (2001),

K. Ermisch et al., Phys. Rev. C 71, 064004 (2005).

17. B. v. Przewoski et al., Phys. Rev. C 74, 064003 (2006).

18. H. R. Amir-Ahmadi et al., Phys. Rev. C 75, 041001 (2007).

19. H. Mardanpour et al., Eur. Phys. J. 31, 383 (2007).

20. E. Stephan et al., Phys. Rev. C 76, 057001 (2007).

21. R. Machleidt and I. Slaus, J. Phys. G 27, R69 (2001).

22. S. A. Coon and H. K. Han, Few-Body Syst. 30, 131 (2001).

23. St. Kistryn et al., Phys. Rev. C 68, 054004 (2003).

24. St. Kistryn et al., Phys. Rev. C 72, 044006 (2005).

25. R. Loren and D. B. Benson, J. Comput. System Sci. 27, 400 (1983).

26. A. Deltuva, A. C. Fonseca and P. U. Sauer, Phys. Rev. C 71, 054005 (2005).

27. H. Witała et al., Phys. Rev. C 83, 044001 (2011).

28. A. Deltuva, A. C. Fonseca and P. U. Sauer, Phys. Rev. C 72, 054004 (2006),

A. Deltuva, A. C. Fonseca and P. U. Sauer, Phys. Rev. C 73, 057001 (2006).

29. H. Witała, J. Golak and R. Skibiński, Phys. Lett. B 634, 374 (2006).

30. St. Kistryn et al., Phys. Lett. B 641, 23 (2006).

31. E. Stephan et al., Phys. Rev. C 82, 014003 (2010).

32. I. Ciepał et al. Phys. Rev. C 85, 017001 (2012).

33. M. Eslami-Kalantari et al., Mod. Phys. Lett. A 24, 839 (2009).

34. H. Mardanpour et al., Phys. Lett. B 687, 14 (2010).

35. H. O. Meyer et al., Phys. Rev. Lett. 93, 112502 (2004).

36. K. Sekiguchi et al., Phys. Rev. C 79, 054008 (2009).

37. A. Ramazani-Moghaddam-Arani et al., Phys. Lett. B 725, 282 (2013). 